\title{
SOLUTION ABOUT A SINGULAR POINT OF A LINEAR DIFFERENTIAL EQUATION INVOLVING A LARGE PARAMETER
}

\author{
BY \\ ROBERT MCKELVEY(1)
}

1. Introduction. The differential equation in question is of the form

$$
\frac{d^{2} u}{d z^{2}}-\left[\lambda^{2} \phi^{2}(z)+\lambda z^{-1} \Psi(z, \lambda)+z^{-2} \tau\right] u=0
$$

considered in a certain bounded domain $R$ about the origin of $z$, under the assumption that $\phi(z)$ does not vanish in $R$ and that the complex parameter $\lambda$ is large in absolute value. The functions $\phi, \Psi$ are bounded and analytic in their arguments when $z \in R$ and $|\lambda|>N . \tau$ is a complex constant.

Asymptotic expansions are given for the solutions of (1), and order estimates are made of the remainder after $n$ terms of the expansion. The approach is classical, based on explicit solution of a special class of equations of type (1). Of the earlier work especial mention should be made of an article [3] by R. E. Langer, whose results pertain to a large class of differential equations, including certain equations of type (1). Also( $\left.{ }^{2}\right)$ the special case $\tau=-3 / 16$ is transformed by the substitution $u(z)=x^{1 / 2} v(x), z=x^{2}$, into a certain case of an equation previously studied by the author [5].

An application of the present results to Laguerre functions is described by Kazarinoff and McKelvey in [2].

In detail, the hypotheses under which Equation (1) is considered are:

(a) $R$ is a simply-connectcd compact subset of the complex plane. The origin is an interior point of $R$.

(b) $\phi(z)$ is a nonvanishing single-valued analytic function in $R\left(^{3}\right)$.

(c) The transformation

$$
\Phi(z)=\int_{0}^{z} \phi(t) d t
$$

is schlicht in $R$, and the image of $R$ under this transformation is convex.

Received by the editors July 29, 1957.

(1) This work was done during 1955-1956 at the Institute for Fluid Dynamics and Applied Mathematics, University of Maryland.

(2) This was pointed out by the referee.

( $\left.{ }^{3}\right)$ The discussion and conclusions which follow remain valid if $\phi(z), \psi(z), \psi_{i}(z)$, and $\tau$ depend in addition upon $\lambda$, being bounded for $|\lambda|>N$, and satisfying the assumptions (b) through (e) for each fixed $\lambda$. See [2]. 
(d) $\Psi(z, \lambda)$ is analytic single-valued for $z \in R$ and at $\lambda=\infty$. Hence, when $|\lambda|>N$ it may be expanded in a termwise-differentiable series

$$
\Psi(z, \lambda)=\psi(z)+\sum_{j=0}^{\infty} \psi_{j}(z) / \lambda^{j+1}
$$

with the coefficients $\psi(z), \psi_{j}(z)$ analytic single-valued functions in $R\left({ }^{4}\right)$.

(e) The equation is normalized (by incorporating a suitable constant factor into $\lambda)$ so that $\phi(0)=1$ and $\operatorname{Re} \psi(0) \geqq 0(5)$.

2. A basic approximating equation. Let

$$
\begin{gathered}
\xi(z, \lambda)=2 \lambda \Phi(z), \\
m=(1+4 \tau)^{1 / 2}, \quad \operatorname{Re}(m) \geqq 0 \\
k(\lambda)=\kappa+\kappa_{0} / \lambda+\kappa_{1} / \lambda^{2}+\cdots+\kappa_{n} / \lambda^{n+1}, \quad n \geqq 0
\end{gathered}
$$

where

$$
\kappa=-\psi(0) / 2
$$

and $\kappa_{1}, \kappa_{2}, \cdots, \kappa_{n}$ are as yet unspecified constants. Let $M(\xi, \lambda)$ be any solution of the Whittaker equation

$$
\frac{d^{2} M}{d \xi^{2}}-\left(\frac{1}{4}-\frac{k(\lambda)}{\xi}-\frac{1-m^{2}}{4 \xi^{2}}\right) M=0,
$$

and consider the function

$$
v(z, \lambda)=\phi^{-1 / 2}(z) M(\xi, \lambda) .
$$

Differentiating twice with respect to $z$,

$$
v^{\prime \prime}(z, \lambda)=4 \lambda^{2} \phi^{3 / 2} \frac{d^{2} M}{d \xi^{2}}+\left(\phi^{-1 / 2}\right)^{\prime \prime} M .
$$

Since both $M, d^{2} M / d \xi^{2}$ can be expressed in terms of $v$ by (3) and (4), we are led immediately to a differential equation for $v$ of the form

$$
\frac{d^{2} v}{d z^{2}}-Q(z, \lambda) v=0
$$

We introduce the quantities

(4) It would be sufficient to require that $\Psi$ be expandable in a Taylor series with remainder, and that the coefficients of the series be merely "sufficiently differentiable." In that case the asymptotic expansion of the solutions could be carried out only to a fixed number of terms.

(5) The normalization $\phi(0)=1$ is unnecessary when $\psi(0)=0$. 


$$
\begin{aligned}
\theta(z) & =\frac{\psi(z)}{z}-\psi(0) \frac{\phi^{2}(z)}{\Phi(z)} \\
\mathrm{X}(z, \lambda) & =\tau\left(\frac{z \phi^{2}}{\Phi^{2}}-\frac{1}{z}\right)+z \phi^{1 / 2}\left(\phi^{-1 / 2}\right)^{\prime \prime}-2 \sum_{j=0}^{n} \frac{\kappa_{j}}{\lambda^{j}} \frac{z \phi^{2}}{\Phi} .
\end{aligned}
$$

Because of the normalization $\phi(0)=1$ (assumption $e$ ), both $\theta(z), X(z, \lambda)$ are analytic for $z \in R$, and at $\lambda=\infty\left(^{4}\right)$. In terms of these quantities,

$$
Q(z, \lambda)=\lambda^{2} \phi^{2}(z)+\lambda\left[\frac{\psi(z)}{z}-\theta(z)\right]+\frac{\tau}{z^{2}}+\frac{\mathrm{X}(z, \lambda)}{z} .
$$

$\mathrm{X}(z, \lambda)$, which is a polynomial in $1 / \lambda$, may be written

$$
\mathrm{X}(z, \lambda)=\sum_{j=0}^{n} \chi_{j}(z) / \lambda^{j}
$$

where every $\chi_{j}(z)$ is analytic in $R$. Note that

$$
\begin{aligned}
& \chi_{0}(0)=\tau \phi^{\prime}(0)-2 \kappa_{0}, \\
& \chi_{j}(0)=-2 \kappa_{j},
\end{aligned} \quad j=1,2, \cdots, n .
$$

These quantities are therefore at our disposal.

We shall designate the wronskian of a pair of functions $f_{1}(z), f_{2}(z)$ by

$$
\text { wron }\left(f_{1}, f_{2} ; z\right)=f_{1}(z) f_{2}^{\prime}(z)-f_{1}^{\prime}(z) f_{2}(z) \text {. }
$$

If $M_{1}, M_{2}$ are two solutions of Equation (3), and $v_{1}, v_{2}$ are the corresponding solutions of Equation (5), computation from (4) shows that

$$
\text { wron }\left(v_{1}, v_{2} ; z\right)=2 \lambda \text { wron }\left(M_{1}, M_{2} ; \xi\right) \text {. }
$$

Hence $v_{1}$ and $v_{2}$ are independent provided $M_{1}$ and $M_{2}$ are. Since neither differential equation has a first derivative term, neither wronskian can depend upon the differentiation variable-both are functions of $\lambda$ alone.

3. A refined approximation. The expression (6) should be compared with the coefficient of $u$ in the given Equation (1). In general the quantities multiplying $\lambda$ in these two expressions are not identical although it may happen that they are so, as for example when $\psi(z) \equiv 0$. In the general case we proceed as follows $\left({ }^{6}\right)$.

Let the functions $a(z), b(z)$ be defined by

$$
\begin{aligned}
& a(z)=\cosh \int_{0}^{z} \theta(t) / 2 \phi(t) d t, \\
& b(z)=[1 / z \phi(z)] \sinh \int_{0}^{z} \theta(t) / 2 \phi(t) d t .
\end{aligned}
$$

( ${ }^{\circ}$ Compare $[3, \S 5]$. 
Both $a(z), b(z)$ are analytic in $R$. Immediately from these definitions,

$$
\begin{aligned}
a^{2}-(z b \phi)^{2} & \equiv 1, \\
2 \phi^{2}(z b)^{\prime}+\left(\phi^{2}\right)^{\prime} z b & =a \theta, \\
2 a^{\prime} & =z b \theta, \quad \text { for } z \in R .
\end{aligned}
$$

Let $v_{1}(z, \lambda), v_{2}(z, \lambda)$ be a linearly independent pair of the functions (4), hence independent solutions of

$$
\frac{d^{2} v}{d z^{2}}-Q(z, \lambda) v=0
$$

Consider the functions

$$
w_{j}(z, \lambda)=a(z) v_{j}(z, \lambda)+\frac{z b(z)}{\lambda} v_{j}^{\prime}(z, \lambda), \quad j=1,2 .
$$

We show first that $w_{1}$ and $w_{2}$ are linearly independent. Differentiating (9) and substituting for $v^{\prime \prime}$ from (5),

$$
\text { wron }\left(w_{1}, w_{2} ; z\right)=D_{0}(z, \lambda) \text { wron }\left(v_{1}, v_{2} ; z\right) \text {, }
$$

where

$$
D_{0}(z, \lambda)=\left|\begin{array}{cc}
a & a^{\prime}+b \frac{z Q}{\lambda} \\
\frac{z b}{\lambda} & a+\frac{(z b)^{\prime}}{\lambda}
\end{array}\right| .
$$

Expending, applying the first of relations (8),

$$
D_{0}(z, \lambda)=1+1 / \lambda\left[(z b)^{\prime} a-z b a^{\prime}-b^{2}\left\{z \psi-z^{2} \theta+\frac{\tau+z \mathrm{X}}{\lambda}\right\}\right] .
$$

The bracketed quantity is analytic for $z \in R, \lambda$ at $\infty$; hence both $D_{0}(z, \lambda)$ and its reciprocal are bounded from zero uniformly for $z \in R,|\lambda|>N$.

The linearly independent functions $w_{1}, w_{2}$ determine a second order linear differential equation of which they are integrals. The computation of the coefficients of this differential equation has been carried out $[5, \S 3]$ for $a(z)$, $z b(z), Q(z, \lambda)$ arbitrary analytic functions for which $D_{0}(z, \lambda)$ is not zero. The result, which applies here when $z \neq 0$, is

$$
\frac{d^{2} w}{d z^{2}}-\frac{D_{0}^{\prime}}{D_{0}} \frac{d w}{d z}-\left[Q+\frac{G_{0}}{D_{0}{ }^{*}}\right] w=0
$$

where 


$$
G_{0}(z, \lambda)=\left|\begin{array}{cc}
a^{\prime \prime}+2(z b)^{\prime} \frac{Q}{\lambda}+z b \frac{Q^{\prime}}{\lambda} & a^{\prime}+z b \frac{Q}{\lambda} \\
2 a^{\prime}+\frac{(z b)^{\prime \prime}}{\lambda} & a+\frac{(z b)^{\prime}}{\lambda}
\end{array}\right| .
$$

Expanding $Q$ as in (6), $\theta$ as in (5a) and applying the relations (8),

$$
G_{0}(z, \lambda)=\lambda \theta+\frac{H_{0}(z, \lambda)}{z}+K_{0}(z)
$$

where

$$
\begin{aligned}
K_{0}(z) & =a(b z)^{\prime} \theta-(b z)(b z)^{\prime \prime} \phi^{2}, \\
H_{0}(z, \lambda) & =\left|\begin{array}{cc}
z a^{\prime \prime}+\frac{2 \tau b^{\prime}}{\lambda}+(z b \Delta)^{\prime}+z b^{\prime} \Delta & z a^{\prime}+b \frac{\tau}{\lambda}+z b \Delta \\
2 a^{\prime}+\frac{(z b)^{\prime \prime}}{\lambda} & a+\frac{(z b)^{\prime}}{\lambda}
\end{array}\right|, \\
\Delta(z, \lambda) & =\psi(0) \frac{z \phi^{2}(z)}{\Phi(z)}+\frac{\mathrm{X}(z, \lambda)}{\lambda} .
\end{aligned}
$$

Evidently $K_{0}(z)$ and $H_{0}(z, \lambda)$ are analytic for $z \in R, \lambda$ at $\infty$.

The transformation

$$
x_{j}=w_{j} D_{0}^{-1 / 2},
$$$$
j=1,2 \text {, }
$$

normalizes Equation (12) to the form

$$
\frac{d^{2} x}{d z^{2}}-S(z, \lambda) x=0
$$

where

$$
\text { (b) } S=Q+\frac{G_{0}}{D_{0}}+\frac{3}{4}\left[\frac{D_{0}^{\prime}}{D_{0}}\right]^{2}-\frac{1}{2} \frac{D_{0}^{\prime \prime}}{D_{0}} \text {. }
$$

Because of specific expressions (11) and (13) for $D_{0}$ and $G_{0}, S(z, \lambda)$ has the form

$$
S(z, \lambda)=\lambda^{2} \phi^{2}+\lambda \frac{\psi(z)}{z}+\frac{\tau}{z^{2}}+\frac{\Omega(z, \lambda)}{z}
$$

where $\Omega(z, \lambda)$ is analytic for $z \in R, \lambda$ at $\infty$. Expression (15) is, of course, to be compared with the coefficient of $u$ in the given Equation (1). 
We write

$$
\Omega(z, \lambda)=\sum_{j=0}^{\infty} \omega_{j}(z) / \lambda^{j}, \quad|\lambda|>N,
$$

in which every $\omega_{j}(z)$ is analytic in $R$. The constant $\kappa_{m}$ with $m=0,1,2, \cdots$, or $n$ comes into the expression for $\Omega(0, \lambda)$ wherever $X(0, \lambda)$ does, hence only in the quantities $Q$ and $G_{0}$ written explicitly in (14b). Moreover, when $X, X^{\prime}$ occur in $G_{0}$ they are invariably multiplied by quantities of the order $1 / \lambda$ or smaller. Consequently $\kappa_{m}$ occurs linearly in $\omega_{m}(0)$, but is absent from every $\omega_{j}(0)$ for which $j<m$. Hence the values of $\omega_{0}(0), \omega_{1}(0), \cdots, \omega_{n}(0)$ are at our disposal.

The solutions $x(z, \lambda)$ of Equation (14) may of course be expressed directly in terms of the solutions of the basic approximating Equation (5):

From (10),

$$
x_{j}(z, \lambda)=D_{0}^{-1 / 2}(z, \lambda)\left[a(z) v_{j}(z, \lambda)+\frac{z b(z)}{\lambda} v_{j}^{\prime}(z, \lambda)\right], \quad j=1,2 .
$$

$$
\text { wron }\left[x_{1}, x_{2} ; z\right]=\text { wron }\left[v_{1}, v_{2} ; z\right] \text {. }
$$

4. The related differential equation. By a process similar to that of the preceding section, we shall next transform equation (14) to obtain one in which the coefficient differs from that of the given Equation (1) by a quantity of the order $1 / \lambda^{n+1}$. Let $\alpha_{0}(z), \eta_{0}(z), \beta_{0}(z)$ be defined by

$$
\begin{aligned}
\alpha_{0} & \equiv 1, \\
\eta_{0} & =\psi_{0}-\omega_{0}, \\
\beta_{0} & =[1 / z \phi(z)] \int_{0}^{z}\left[\eta_{0}(s) / 2 s \phi(s)\right] d s
\end{aligned}
$$

$\omega_{0}(0)$, until now unspecified, is chosen equal to $\psi_{0}(0)$. Hence $\eta_{0}(z)$ vanishes at the origin and $\beta_{0}(z)$ is defined and analytic throughout $R$.

Let $\alpha_{1}(z), \eta(z), \beta_{1}(z)$ be defined by

$$
\begin{aligned}
& \alpha_{1} \equiv 0, \\
& \eta_{1}=\left(\psi_{1}-\omega_{1}\right)-\psi \beta_{0}-z\left(2 \psi \beta_{0}^{\prime}-\psi^{\prime} \beta_{0}\right), \\
& \beta_{1}=[1 / z \phi(z)] \int_{0}^{z}\left[\eta_{1}(s) / 2 s \phi(s)\right] d s .
\end{aligned}
$$

Choosing $\omega_{1}(0)$ so that $\eta_{1}(z)$ vanishes at the origin, $\beta_{1}(z)$ is defined and analytic throughout $R$.

In this fashion a sequence of functions is defined. At the $\nu$ th stage, and in terms of previously determined quantities, let $\alpha_{\nu}(z), \eta_{\nu}(z), \beta_{\nu}(z)$ be defined by 


$$
\begin{aligned}
\alpha_{\nu}= & -\frac{1}{2} \int_{0}^{z}\left[\left(s \beta_{\nu-2}\right)^{\prime \prime}+\sum_{j=0}^{\nu-2}\left(\psi_{\nu-j-2}-\omega_{\nu-j-2}\right) \beta_{j}\right] d s \\
\eta_{\nu}= & \sum_{j=0}^{\nu}\left(\psi_{\nu-j}-\omega_{\nu-j}\right) \alpha_{j}-\psi \beta_{\nu-1}-z\left(2 \psi \beta_{\nu-1}^{\prime}+\psi^{\prime} \beta_{\nu-1}\right) \\
& -2 \tau \beta_{\nu-2}^{\prime}-z \alpha_{\nu}^{\prime \prime}-\sum_{j=0}^{\nu-2}\left[\omega_{\nu-j-2} \beta_{j}+z\left(2 \omega_{\nu-j-2} \beta_{j}^{\prime}+\omega_{\nu-j-2}^{\prime} \beta_{j}\right)\right], \\
\beta_{\nu}= & {[1 / z \phi(z)] \int_{0}^{z}\left[\eta_{\nu}(s) / 2 s \phi(s)\right] d s . }
\end{aligned}
$$

$\omega_{\nu}(0)$ is to be chosen so that $\eta_{\nu}(z)$ vanishes at the origin. Hence $\alpha_{\nu}(z), \eta_{\nu}(z)$, $\beta_{\nu}(z)$ are all analytic in $R$.

The sequence is terminated with the stage $\nu=n$. At this stage, all the quantities $\kappa_{0}, \kappa_{1}, \cdots, \kappa_{n}$ have been determined. We write

$$
\begin{aligned}
& A(z, \lambda)=\sum_{j=0}^{n} \alpha_{j}(z) / \lambda^{j}, \quad B(z, \lambda)=\sum_{j=1}^{n} \beta_{j}(z) / \lambda^{j} \\
& D_{1}(z, \lambda)=\left|\begin{array}{cc}
A & A^{\prime}+z B \frac{S}{\lambda^{2}} \\
\frac{z B}{\lambda^{2}} & A+\frac{(z B)^{\prime}}{\lambda^{2}}
\end{array}\right| .
\end{aligned}
$$

By inspection, $D_{1}(z, \lambda)$ is analytic for $z \in R, \lambda=\infty$. Further, $D_{1}(z, \lambda)$ differs from unity by a quantity of order $1 / \lambda^{2}$ (since $\left.\alpha_{0} \equiv 1, \alpha_{1} \equiv 0\right)$; hence both $D_{1}$ and its reciprocal are bounded from zero when $|\lambda|>N$.

Proceeding in a manner analogous to $\S 3$, one may show $\left({ }^{7}\right)$ that the functions

$$
y_{j}(z, \lambda)=D_{1}^{-1 / 2}(z, \lambda)\left[A(z, \lambda) x_{j}(z, \lambda)+\frac{z B(z, \lambda)}{\lambda^{2}} x_{j}^{\prime}(z, \lambda)\right], \quad j=1,2
$$

for which

$$
\text { wron }\left[y_{1}, y_{2} ; z\right]=\text { wron }\left[x_{1}, x_{2} ; z\right] \text {, }
$$

determine a linear differential equation of the form

$$
\frac{d^{2} y}{d z^{2}}-\left[\lambda^{2} \phi^{2}(z)+\lambda \frac{\Psi(z, \lambda)}{z}+\frac{\tau}{z^{2}}-\frac{\Theta(z, \lambda)}{\lambda^{n+1} z}\right] y=0
$$

( ${ }^{7}$ ) Apart from notational differences the computation is formally identical with $[3, \S 6]$, which is meaningful here since, with the adjustments of $\omega_{j}(0)$ explained above, all quantities involved are well defined. That the error term in (22) has a simple (rather than double) pole at $z=0$ is evident on making the substitutions $A, B / \lambda, D_{1}, S$ for $a, b, D_{0}, Q$ in (12), (14) and expanding $S$ as in (15). 
where $\Theta(z, \lambda)$ is analytic for $z \in R, \lambda=\infty$.

By combining the expressions (4), (9), and (16), we may express a pair of solutions of the related equation directly in terms of the corresponding solutions of the Whittaker equation in the form

$$
y_{j}(z, \lambda)=E(z, \lambda) M_{j}(\xi, \lambda)+\lambda^{-1} F(z, \lambda)\left[\xi \frac{d}{d \xi} M_{j}(\xi, \lambda)\right]
$$

where the functions $E(z, \lambda), F(z, \lambda)$ are analytic for $z \in R, \lambda=\infty$.

Differentiating (23) by $z$ and substituting for $d^{2} M_{j} / d \xi^{2}$ from the Whittaker equation, we obtain $y_{j}(z, \lambda)$ in the form

$$
y_{j}^{\prime}(z, \lambda)=E^{*}(\xi, \lambda) M_{j}(\xi, \lambda) / \xi+\lambda F^{*}(z, \lambda) d / d \xi M_{j}(\xi, \lambda)
$$

with $E^{*}, F^{*}$ analytic for $z \in R, \lambda=\infty$.

Finally, from (7), (17), and (21),

$$
\text { wron }\left[y_{1}, y_{2} ; z\right]=2 \lambda \text { wron }\left[M_{1}, M_{2} ; \xi\right] \text {. }
$$

5. Solutions of the related equation. The standard $\left.{ }^{8}\right)$ Whittaker function $\mathfrak{M}_{k(\lambda), m / 2}(\xi)$ has for $|\lambda|>N,|\xi| \leqq M$, where $M$ is a large (fixed) positive number, the structure

$$
\begin{aligned}
\mathfrak{T T}_{k(\lambda), m / 2}(\xi) & =\xi^{m+1 / 2} O(1), \\
\frac{d \mathfrak{T}_{k(\lambda), m / 2}(\xi)}{d \xi} & =\xi^{m-1 / 2} O(1) ;
\end{aligned}
$$

it is the solution of (3) which vanishes to the highest order at the origin. Since

$$
\text { wron }\left[W_{k(\lambda), m / 2}, \mathfrak{T}_{k(\lambda), m / 2} ; \xi\right]=1 / \Gamma\left(\frac{1+m}{2}-k(\lambda)\right) \text {, }
$$

therefore the standard function $W_{k(\lambda), m / 2}(\xi)$ provides a second (independent) solution of the Whittaker equation for the range

$$
\begin{aligned}
\operatorname{Re}(m) & \geqq 0, \\
\operatorname{Re}(k(\lambda)) & \leqq \epsilon, \quad 0<\epsilon<1 / 2
\end{aligned}
$$

to which the parameters are restricted when $|\lambda|>N$, by the normalization of $\S 1$. We have, respectively, for $m=0, m=1$, or otherwise, that

$$
\begin{array}{rlrl}
W_{k(\lambda), m / 2}(\xi) & =\xi^{1 / 2}[\ln \xi \cdot O(1)+O(1)], & & O(1), \text { or } \xi^{-m+1 / 2} O(1), \\
\frac{d W_{k(\lambda), m / 2}(\xi)}{d \xi} & =\xi^{-1 / 2}[\ln \xi \cdot O(1)+O(1)], & \frac{\ln \xi}{\Gamma(-k)}+O(1), \text { or } \xi^{-m-1 / 2} O(1)
\end{array}
$$

when $|\lambda|>N,|\xi| \leqq M$.

${ }^{(8)}$ Notation and formulae quoted in this section are from Buchholz [1]. The symbol $O(1)$ designates always a function which is analytic as well as bounded; the symbol $O(\lambda)$ means $\lambda O(1)$, and so on. 
Whittaker functions of particularly simple structure for large $\xi$ are the functions

$$
W_{\sigma k(\lambda), m / 2}\left(\xi e^{-\nu \pi i}\right)
$$

where $\sigma=(-1)^{\nu}$, and $\nu$ is any integer. When $\xi$ falls in the range

$$
\Sigma_{\nu}:|\xi|>M ; \quad(\nu-3 / 2) \pi+\epsilon_{1} \leqq \arg \xi \leqq(\nu+3 / 2) \pi-\epsilon_{1} .
$$

with $\epsilon_{1}$ a small fixed positive number, then both $W_{\sigma k(\lambda), m / 2}\left(\xi e^{-\boldsymbol{\nu} \pi \boldsymbol{i}}\right)$ and the $\xi$ derivative of this function are of the structure $\xi^{\sigma k} e^{-\sigma \xi / 2} O(1)$. The $W$-functions corresponding to consecutive integers are independent, with wronskian given by the formula

$$
\text { wron }\left[W_{\sigma k, m / 2}\left(\xi e^{-\nu \pi i}\right), W_{-\sigma k, m / 2}\left(\xi e^{-(\nu \pm 1) \pi i}\right) ; \xi\right]=\sigma e^{ \pm \sigma k \pi i} .
$$

We remark finally that when $\arg \xi$ is unrestricted and $|\xi|>M$, the function $\mathfrak{T}_{k, m / 2}(\xi)$, together with its derivative, is of the structure $\xi^{-k} e^{1 / 2 \xi} O(1)$ $+\xi^{k} e^{-1 / 2 \xi} O(1)$. The same is true of the functions $W_{o k, m / 2}\left(\xi e^{-\nu \pi i}\right)$.

The solutions of the related equation which correspond by (23) to the Whittaker functions

$$
\mathfrak{M}_{k(\lambda), m / 2}(\xi), \quad W_{\sigma k(\lambda), m / 2}\left(\xi e^{-\nu \pi i}\right)
$$

are denoted by

$$
y_{*}(z, \lambda), \quad y_{\nu}(z, \lambda) \text {. }
$$

When $|\xi| \leqq M$, the function $y_{*}(z, \lambda)$ has the structure

$$
\begin{aligned}
y_{*}(z, \lambda) & =\xi^{(m+1) / 2} O(1), \\
\frac{d y_{*}(z, \lambda)}{d z} & =\xi^{(m-1) / 2} O(\lambda),
\end{aligned}
$$

since when $\lambda$ is fixed, $\xi$ is of the order of $z$ near $z=0$. Therefore $y_{*}$ is the solution of larger exponent of the related equation. The (independent) function $y_{0}(z, \lambda)$ has the structure, for $m=0, m \neq 0$ respectively

$$
\begin{aligned}
y_{0}(z, \lambda) & =\xi^{1 / 2}[\ln \xi \cdot O(1)+O(1)], \quad \xi^{-m+1 / 2} O(1) ; \\
\frac{d y_{\mathfrak{\imath}}(z, \lambda)}{d z} & =\xi^{-1 / 2}[\ln \xi \cdot O(\lambda)+O(\lambda)], \quad \xi^{-m-1 / 2} O(\lambda),
\end{aligned}
$$

when $|\xi| \leqq M$.

Finally, when $\xi \in \Sigma_{\nu}$, then

$$
\begin{aligned}
y_{\nu}(z, \lambda) & =\xi^{\sigma k(\lambda)} e^{-\sigma \xi / 2} O(1), \\
\frac{d y_{\nu}(z, \lambda)}{d z} & =\xi^{\sigma k(\lambda)} e^{-\sigma \xi / 2} O(\lambda),
\end{aligned}
$$


and when $|\arg \xi|<\pi / 2,|\xi|>M$, then

$$
\begin{aligned}
y_{*}(z, \lambda) & =\xi^{-k(\lambda)} e^{\xi / 2} O(1), \\
\frac{d y_{*}(z, \lambda)}{d z} & =\xi^{-k(\lambda)} e^{\xi / 2} O(\lambda) .
\end{aligned}
$$

From Equation (25),

$$
\text { wron }\left[y_{*}, y_{0}\right]=2 \lambda / \Gamma\left(\frac{1+m}{2}-k\right) ; \quad \text { wron }\left[y_{\nu}, y_{\nu \pm 1}\right]=2 \lambda \sigma e^{ \pm \sigma k \pi i}
$$

so that either wronskian is $O(\lambda)$, and its reciprocal is $O(1 / \lambda)$.

6. Estimates of the solutions. Let $y(z, \lambda)$ be any solution of the related equation, and $z_{0}(\lambda)$ a point of $R$. Consider the integral equation $\left({ }^{9}\right)$

$$
u(z)=y(z)+\int_{z_{0}}^{z} \frac{y(z) \tilde{y}(t)-\tilde{y}(z) y(t)}{\lambda^{n+1} \text { wron }[y, \tilde{y}]} \frac{\Theta(t)}{t} u(t) d t
$$

in which $\tilde{y}(z, \lambda)$ is a second independent solution of the related equation, and the integration is along a sectionally smooth contour in $R$. It is familiar $\left({ }^{9}\right)$ that a solution $u(z)$ of (30) is also a solution of the given Equation (1). Here $u(z)$ depends upon $y(z)$ and $z_{0}$, not at all upon the choice of $\tilde{y}$, which may therefore be taken as different functions along different portions of the contour. Differentiating (30) leads to an expression for $u^{\prime}(z)$ :

$$
u^{\prime}(z)=y^{\prime}(z)+\int_{z_{0}}^{z} \frac{y^{\prime}(z) \tilde{y}(t)-\tilde{y}^{\prime}(z) y(t)}{\lambda^{n+1} \operatorname{wron}[y, \tilde{y}]} \frac{\Theta(t)}{t} u(t) d t .
$$

Higher derivatives may be obtained directly from the differential equation.

Estimates of various functions $u(z)$ will be based upon the

Lemma. If, for $z$ and $t$ in a simply-connected region $R^{*}(\lambda)$, the functions $f(z, \lambda)$ and $K(z, t, \lambda)$ are analytic in $z$ and in $t$ and, with $\Gamma(z, \lambda)$ a specific path from $z_{0} \in \bar{R}^{*}$ to $z$, exists and approaches 0 uniformly as $\lambda \rightarrow \infty$, then when $|\lambda|>N$ the integral equation

$$
g(z, \lambda)=f(z, \lambda)+\int_{z_{0}}^{z} K(z, t, \lambda) g(t, \lambda) d t
$$

has in $R^{*}(\lambda)$ a solution $g(z, \lambda)$, and, provided $|f(z, \lambda)|$ is bounded,

$$
g(z, \lambda)=f(z, \lambda)+O(\|K\|) \text { uniformly in } \lambda .
$$

To prove the lemma we have only to consider the Neumann expansion $g=f+K f+K^{2} f \cdots$. Since $\left|K^{n} f\right| \leqq\|K\|^{n} \cdot|f|$, therefore the expansion con-

(9) Compare e.g. [4]. For concise notation, the dependence upon $\lambda$ of certain functions has been suppressed. 
verges when $|\lambda|>N$, defining $g$. The quantity $g-f$ is dominated by the series $\left(\|K\|+\|K\|^{2}+\cdots\right)|f|$, and hence is $O(\|K\|)$.

The lemma usually cannot be applied directly to (30), but the latter expression will always be rewritten in such form that the lemma does apply.

7. The solution of higher index at the origin. Let $y(z), \tilde{y}(z)$ in (30) be respectively the functions $y^{*}(z), y_{0}(z)$ defined in $\$ 5$. We restrict attention to values of $z, \lambda$ such that $|\xi(z, \lambda)| \leqq M$ and integrate from $z_{0}=0$ along a curve $\arg \xi=$ constant.

Supposing for the present that $m \neq 0$, let the functions $Y(z), \tilde{Y}(z), U(z)$ be defined by

$$
\begin{aligned}
& y(z)=\xi^{(m+1) / 2} Y(z), \\
& \tilde{y}(z)=\xi^{(-m+1) / 2} \widetilde{Y}(z), \\
& u(z)=\xi^{(m+1) / 2} U(z) .
\end{aligned}
$$

Because of (26) and (27) it is evident that $Y(z), \tilde{Y}(z)$ are bounded functions of $z$ and $\lambda$ when $|\xi| \leqq M$.

Equation (30) may be rewritten in terms of the above functions as

$$
U(z)=Y(z)+\int_{z_{0}}^{z} \frac{Y(z) \tilde{Y}(t)-\tilde{Y}(z) Y(t) \Pi(t, z)}{\lambda^{n} \text {. wron }[y, \tilde{y}]} C(t, \lambda) U(t) d t
$$

where

and

$$
\Pi(t, z)=[\xi(t) / \xi(z)]^{m}
$$

$$
C(t, \lambda)=\frac{\xi(t) \Theta(t, \lambda)}{\lambda t} \text {. }
$$

We consider the expression

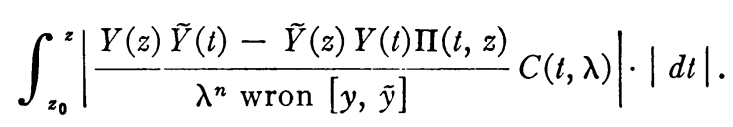

Since on the contour $\xi(t) / \xi(z)$ is positive and $\leqq 1$, therefore $\Pi(t, z)$ is bounded. Also $\xi(t)$ is of the order $\lambda t$ near $t=0$, so $C(t, \lambda)$ is likewise bounded. It follows that the expression (33) is $O\left(\xi / \lambda^{n+2}\right)$. Hence, by the lemma, the integral equation (32) has a solution $U(z)$ and $U(z)=Y(z)+0\left(\xi / \lambda^{n+2}\right)$. From this follows

THEOREM 1. When $m \neq 0$, there is a solution of the given differential equation, we designate it by $u_{*}(z)$, such that when $|\xi| \leqq M,|\lambda|>N$,

$$
u_{*}(z)=y_{*}(z)+\xi^{(m+3) / 2} O\left(1 / \lambda^{n+2}\right),
$$

and (computing from (31))

$$
u_{*}^{\prime}(z)=y_{*}^{\prime}(z)+\xi^{(m+1) / 2} O\left(1 / \lambda^{n+1}\right) .
$$


Evidently $u(z)$ is distinguished as the solution of higher index at the origin.

When $m=0 /$ the computations must be somewhat modified. Supposing first that $|\xi| \leqq \gamma<1$ for some fixed number $\gamma$, we define $\tilde{Y}(z)$ by

$$
\tilde{y}(z)=\xi^{1 / 2} \ln \xi \cdot \tilde{Y}(z)
$$

and $Y(z), U(z)$ as before. Both $Y(z), \tilde{Y}(z)$ are bounded when $|\xi| \leqq \gamma$. Once again (30) is rewritten in the form (32) where now

$$
\Pi(t, z)=\ln \xi(z) / \ln \xi(t)
$$

and

$$
C(t, \lambda)=\ln \xi(t) \cdot \frac{\xi(t) \Theta(t)}{\lambda t} .
$$

Hence (33) is now of the order $O\left(\xi \ln \xi / \lambda^{n+1}\right)$ and

$$
u(z)=y(z)+\xi^{3 / 2} \ln \xi O\left(1 / \lambda^{n+2}\right) .
$$

Second, when $\gamma<|\xi(z)| \leqq M$, there is a single point $z_{1}$ on the contour such that $\left|\xi\left(z_{1}\right)\right|=\gamma$. We write (30) in its original form and consider separately the integration from 0 to $z_{1}$ and the integration from $z_{1}$ to $z$. Since we have already an estimate of $u(t)$ over the former range, we may compute directly that the corresponding integral is of the order $O\left(1 / \lambda^{n+2}\right)$. If now this term is incorporated into the function $f(z, \lambda)$ of the lemma, one may conclude that

$$
u_{*}(z)=y_{*}(z)+O\left(1 / \lambda^{n+2}\right) .
$$

Combining this with the previous expression we have proved

THEOREM 2. When $m=0$, the given equation possesses a solution, designated $u_{*}(z)$, such that when $|\xi| \leqq M,|\lambda|>M$

$$
u_{*}(z)=y_{*}(z)+\xi^{3 / 2}\left[\ln \xi \cdot O\left(1 / \lambda^{n+2}\right)+O\left(1 / \lambda^{n+2}\right)\right]
$$

and, from (31),

$$
u_{*}^{\prime}(z)=y_{*}^{\prime}(z)+\xi^{1 / 2}\left[\ln \xi \cdot O\left(1 / \lambda^{n+1}\right)+O\left(1 / \lambda^{n+1}\right)\right] .
$$

8. Solutions of simple structure when $|\xi|$ is large. We now restrict attention to values of $z, \lambda$ for which $\xi \in \Sigma_{\nu}(\S 5)$. In the expression (30) let $z_{0}(\lambda)$ be a point at which $\operatorname{Re}(\sigma \xi)$ assumes its maximum value in $\Sigma_{\nu}$. The integration, as viewed in the image region $\Sigma_{\nu}$, is taken along a straight line, or when necessary, along a pair of straight lines joined by an arc of $|\xi|=M$, it being required that $\operatorname{Re}(\sigma \xi)$ be monotone decreasing on the linear part of such a path from $z_{0}(\lambda)$ to $z$. The role of $y(z)$ is taken by the function $y_{v}(z)$. The role of $\tilde{y}(z)$ is taken by $y_{\nu+1}(z)$ on a portion of the contour for which $\arg \xi>\nu \pi$, and by $y_{\nu-1}(z)$ when $\arg \xi<\nu \pi$. We define $Y(z), \tilde{Y}(z), U(z)$ by 


$$
\begin{aligned}
& y(z)=\xi^{\sigma k} e^{-\sigma \xi / 2} Y(z), \\
& \tilde{y}(z)=\xi^{-\sigma k} e^{\sigma \xi / 2} \tilde{Y}(z), \\
& u(z)=\xi^{\sigma k} e^{-\sigma \xi / 2} U(z) .
\end{aligned}
$$

Evidently both $Y(z), \tilde{Y}(z)$ are bounded when $\xi \in \Sigma_{\nu}$. In terms of the newly defined functions, the expression (30) may again be put in the form (32) where now

$$
\begin{aligned}
& \Pi(t, z)=\left[\frac{\xi(t)}{\xi(z)}\right]^{2 \sigma k} e^{-\sigma[\xi(t)-\xi(z)]}, \\
& C(t, \lambda)=\frac{\Theta(t, \lambda)}{\lambda t} .
\end{aligned}
$$

$\Pi(t, z)$ is bounded uniformly on the class of contours being considered. Hence the expression (33) is $O\left(\ln \lambda / \lambda^{n+2}\right)$ and we have the

THEOREM 3. There is a solution $u_{v}(z)$ of the given differential equation such that when $\xi \in \Sigma_{\nu},|\lambda|>N$, then

$$
\begin{aligned}
u_{\nu}(z) & =y_{\nu}(z)+\xi^{\sigma k} e^{-\sigma \xi / 2} O\left(\ln \lambda / \lambda^{n+2}\right), \\
u_{\nu}^{\prime}(z) & =y_{\nu}^{\prime}(z)+\xi^{\sigma k} e^{-\sigma \xi / 2} O\left(\ln \lambda / \lambda^{n+1}\right) .
\end{aligned}
$$

9. A second solution when $|\xi| \leqq M$. We now investigate the structure of the solution $u_{0}(z)$, defined above, when $z, \lambda$ are such that $|\xi| \leqq M$. The functions $y, \tilde{y}$ in (30) are taken to be respectively $y_{0}$ and $y_{*}$. The integration contour is composed of a segment of a curve arg $\xi=$ constant, from the point $z_{0}$ (defined in $\S 8$ above) to a point $z_{1}$ on $|\xi|=M$, and of a spiral into the region $|\xi| \leqq M$, from $z_{1}$ to the point $z$. (We shall assume that arg $z$ varies boundedly so that the spiral is of bounded length.)

Suppose first that $m \neq 0$. We must consider separately the integration from $z_{0}$ to $z_{1}$ and the integration from $z_{1}$ to $z$. With (30) written in its original form, the former integral involves only known functions, and is of the structure

$$
\begin{gathered}
\int_{z_{0}}^{z}\left[\xi(z)^{(-m+1) / 2} \xi(t)^{-k} e^{\xi(t) / 2} O(1)+\xi(z)^{(m+1) / 2} \xi(t)^{k} e^{-\xi(t) / 2} O(1)\right] \frac{\xi(t)^{k} e^{-\xi(t) / 2} O(1)}{\lambda^{n+2} t} d t \\
=\frac{\xi(z)^{(-m+1) / 2}}{\lambda^{n+2}} \int_{z_{0}}^{z}\left[O(1)+\xi^{m}(z) \xi(t)^{2 k} e^{-\xi(t)} O(1)\right] \frac{d t}{t} .
\end{gathered}
$$

In this expression, both $\xi^{m}(z)$ and $\xi^{2 k}(t) e^{-\xi(t)}$ are bounded: the former because $|\xi(z)| \leqq M$, and the latter because $\operatorname{Re} \xi(t)>0$. Hence the integral shown is $O(\ln \lambda)$, and (30) becomes

$$
\begin{aligned}
u(z)= & y(z)+\xi^{-m+1 / 2} O\left(\ln \lambda / \lambda^{n+2}\right) \\
& +\int_{z_{1}}^{z} \frac{y(z) \tilde{y}(t)-\tilde{y}(z) y(t)}{\lambda^{n+1} \text { wron }[y, \tilde{y}]} \frac{\Theta(t)}{t} u(t) d l .
\end{aligned}
$$


The lemma is now applied to Equation (37); the details are almost identical with those described in $\$ 7$. It is found that

$$
u(z)=y(z)+\xi^{-m+1 / 2} O\left(1 / \lambda^{n+1}\right) .
$$

When $m=0$, the procedure just outlined must be somewhat modified, but no special difficulties arise. The results are described as

Theorem 4. When $|\xi| \leqq M,|\lambda|>N$, and when $m \neq 0$, then

$$
\begin{aligned}
\text { (a) } u_{0}(z) & =y_{0}(z)+\xi^{(-m+1) / 2} O\left(1 / \lambda^{n+1}\right), \\
\text { (b) } u_{0}^{\prime}(z) & =y_{0}^{\prime}(z)+\xi^{(-m-1) / 2} O\left(1 / \lambda^{n}\right) .
\end{aligned}
$$

When, on the other hand, $m=0$, then

$$
\begin{aligned}
u_{0}(z) & =y_{0}(z)+\xi^{1 / 2}\left[\ln \xi \cdot O\left(1 / \lambda^{n+1}\right)+O\left(1 / \lambda^{n+1}\right)\right], \\
u_{0}^{\prime}(z) & =y_{0}^{\prime}(z)+\xi^{-1 / 2}\left[\ln \xi \cdot O\left(1 / \lambda^{n}\right)+O\left(1 / \lambda^{n}\right)\right] .
\end{aligned}
$$

10. The structure of $u_{*}$ when $|\xi|$ is large. This proceeds such as in $\S 9$. The integration contour from the origin to a point $Z$ for which $|\xi|>M$ is chosen to be a curve $\arg \xi=$ constant. The point on this curve for which $|\xi|=M$ is designated by $Z_{1}$. On the portion of the curve between 0 and $Z_{1}$ the functions $y, \tilde{y}$ are taken to be $y_{0}$ and $y_{*}$. All quantities involved in this integral have been previously estimated so that the integral itself is found to be (for $m \neq 0$ ) of the order

$$
\xi^{k} e^{-\xi / 2} O\left(1 / \lambda^{n+1}\right)+\xi^{-k} e^{\xi / 2} O\left(1 / \lambda^{n+1}\right) .
$$

The computations are similar to $\$ 7$.

Now let us assume that

$$
\pi(\nu-1 / 2) \leqq \arg \xi \leqq \pi(\nu+1 / 2) .
$$

On the portion of the contour between $Z_{1}$ and $Z$ the role of $y$ is assigned to $y_{\nu}$ and that of $\tilde{y}$ to $y_{\nu+1}$ if $\arg \xi>\nu \pi$ or to $y_{\nu-1}$ if $\arg \xi<\nu \pi$. Proceeding as in $\S 8$, we may now estimate $u_{*}$. The following result is valid regardless of the value of $\arg \xi$ or of $m$.

Theorem 5. When $|\xi| \geqq M$ and $|\lambda|>N$ then

$$
u_{*}(z)=y_{*}(z)+\xi^{k} e^{-\xi / 2} O\left(1 / \lambda^{n+1}\right)+\xi^{k} e^{\xi / 2} O\left(1 / \lambda^{n+1}\right)
$$

and

$$
u_{*}^{\prime}(z)=y_{*}^{\prime}(z)+\xi^{k} e^{-\xi / 2} O\left(1 / \lambda^{n}\right)+\xi^{k} e^{\xi / 2} O\left(1 / \lambda^{n}\right) .
$$

\section{BIBLIOGRAPHY}

1. H. Buchholz, Die Konfluente Hypergeometrische Function, Springer-Verlag, Berlin, 1953.

2. N. D. Kazarinoff and R. W. McKelvey, A note on the asymptotic behavior of Laguerre polynomials and functions for large n, Bull. Amer. Math. Soc. Abstract 62-6-677.

3. R. E. Langer, The asymptotic solutions of ordinary linear differential equations of the 
second order, with special reference to a turning point, Trans. Amer. Math. Soc. vol. 67 (1949) pp. 461-490.

4. - On the asymptotic solutions of ordinary differential equations, with reference to the Stokes' phenomenon about a singular point, Trans. Amer. Math. Soc. vol. 37 (1935) pp. 397416.

5. R. W. McKelvey, The solutions of second order linear ordinary differential equations about a turning point of order two, Trans. Amer. Math. Soc. vol. 79 (1955) pp. 103-123.

University of Colorado,

Boulder, Colo. 